\title{
Construction of potentials with multiple spectral singularities
}

\author{
Vladimir V. Konotop ${ }^{1,2}$ and Dmitry A. Zezyulin ${ }^{3}$ \\ ${ }^{1}$ Centro de Física Teórica e Computacional Universidade de Lisboa, Campo Grande, \\ Edifício C8, Lisboa 1749-016, Portugal \\ ${ }^{2}$ Departamento de Física, Faculdade de Ciências, Campo Grande, Edifício C8, Lisboa \\ 1749-016, Portugal \\ ${ }^{3}$ ITMO University, St. Petersburg 197101, Russia \\ E-mail: dzezyulin@itmo.ru
}

\begin{abstract}
We develop an approach for designing complex potentials with two or three coexisting spectral singularities in the spectra of the respective Schrödinger operators. The approach is illustrated with several examples. In addition, we offer a simple recipe to create a second-order spectral singularity by intentionally colliding two simple ones.
\end{abstract}

\section{Introduction}

Spectral singularities (SSs) in spectra of non-Hermitian operators is a long-standing subject in mathematics pioneered about sixty years ago [1. Independently, in physical literature 2] of approximately the same years various effects were reported that are presently known to be originated by SSs. These are the effects of coherent perfect absorption (alias CPA or antilasing) and lasing. About a decade ago, the interest in SSs was regained chiefly due to a series of theoretical studies [3, 4, 5, 6, 7] where the relation between SSs and physical phenomena of absorption and lasing was clarified, and experiments on coherent perfect absorption of electromagnetic waves [8]. For a more detailed discussion of the role that SSs play in scattering properties of physical systems see reviews [9].

In order to obtain a SS in a spectrum of a non-Hermitian Schrödinger operator, the shape of the underlying complex potential needs to be tuned precisely, i.e. by far not every complex potential supports a SSs. Therefore the presence of SS is a quite demanding property that requires a sharp adjustment of the physical system. At the same time, a number of complex potentials with SSs at a given wavelength is infinite. In the one-dimensional setting, to which our discussion will be limited, such potentials can be constructed using different algorithms [10, 11]. Moreover, it has been shown in [12], that all complex potentials of the stationary one-dimensional Schrödinger equation with SSs in their spectra have a universal analytical form. This last feature 
yields an alternative way of constructing potentials, either with on a finite support or distributed over the whole real axis, that feature a SS at a given wavenumber $k_{0}$.

It is also known, that a spectrum of a given potential may have more than one SSs. In particular, this is the case when both $k_{0}$ and $-k_{0}$ happen to be SSs simultaneously. This situation is typical for parity-time $(\mathcal{P} \mathcal{T}$-)symmetric systems [7. Such SSs are often termed self-dual [13] and represent an especially appealing physical situation when the same system can operate as a laser or a CPA for waves of the same length. Self-dual spectral singularities are also known to exist in complex potentials with other antilinear symmetries [12, 14] and in potentials without $\mathcal{P} \mathcal{T}$ symmetry [13]. An alternative way to obtain a pair of SSs in the spectrum consists in firstly creating a single second-order SS and then splitting it into two first-order ones by a small perturbation [11, 15]. In this scenario, the emerging SSs will have close wavenumbers. Some exactly solvable models with multiple spectral singularities are also known [16, 17]. At the same time, there is an open general question about a systematic approach to construction of complex potentials having two or more SSs at arbitrary prescribed wavenumbers. In this paper we offer a solution to this problem.

The rest of this paper is organized as follows. In section 2 we present a general approach to construction of potentials with several SSs. In section 3 we apply the approach to construct potentials with two coexisting SSs. In section 4 we demonstrate how our method can be used to generate a higher-order SS by colliding two first-order SSs. In section 5 we discuss peculiarities of the approach in a situation when the potential has a special antilinear symmetry, i.e. a property of pseudo-Hermiticity. In section $[$ we construct a potential with three spectral singularities. Section 7 concludes the paper.

\section{Construction of potentials with several spectral singularities}

Consider a normalized one-dimensional stationary Schrödinger equation

$$
-\psi^{\prime \prime}+U(x) \psi=k^{2} \psi
$$

on the whole axis, $x \in \mathbb{R}$, where $\psi=\psi(x)$ is a complex-valued wavefunction, $U(x)$ is a complex-valued potential vanishing at the infinity: $\lim _{x \rightarrow \pm \infty} U(x)=0, k$ is a spectral parameter (dimensionless wavenumber), and a prime stands for the derivative with respect to $x$. The starting point of our approach is the sufficient condition for the potential $U(x)$ to have a spectral singularity [12, section 3]. Assume that the potential $U(x)$ admits the representation

$$
U(x)=-w_{0}^{2}(x)-i w_{0}^{\prime}(x)+k_{0}^{2},
$$

where $k_{0}$ is a nonzero real, and the base function $w_{0}(x)$ has an asymptotic behavior

$$
\begin{aligned}
& w_{0}(x)=k_{0}+w_{-\infty}(x), \quad w_{-\infty}=O\left(|x|^{-2}\right) \quad \text { at } x \rightarrow-\infty, \\
& w_{0}(x)=-k_{0}+w_{\infty}(x), \quad w_{\infty}=O\left(|x|^{-2}\right) \quad \text { at } x \rightarrow \infty .
\end{aligned}
$$


Then the potential $U(x)$ has a SS at $k=k_{0}$, and the solution of (1) associated with this SS (i.e., SS-solution) reads 12

$$
\psi_{0}(x)=\rho \exp \left[-i \int_{x_{0}}^{x} w_{0}(\xi) d \xi\right]
$$

where $x_{0}$ is any point of the real axis, and $\rho$ is a nonzero constant. Indeed, it is easy to check that there exist nonzero constants $\rho_{ \pm}$such that $\lim _{x \rightarrow \pm \infty}\left[\psi_{0}(x)-\rho_{ \pm} e^{ \pm i k_{0} x}\right]=0$, which means that solution $\psi_{0}$ satisfies purely outgoing (for $k_{0}>0$ ) or purely incoming (for $k_{0}<0$ ) wave boundary conditions, i.e. corresponds to the lasing mode or to the CPA-operation (antilaser), respectively.

Equations (2) and (5) presuppose that the function $w_{0}(x)$ is sufficiently wellbehaved. However, the discussion can be generalized easily on the situation when $w_{0}(x)$ has a finite number of discontinuities at points $x_{1}<x_{2}<\ldots<x_{N}$, provided that $w_{0}(x)$ behaves as $w_{0}(x)=i\left(x-x_{j}\right)^{-1}+O(1)_{x \rightarrow x_{j}}$ around each discontinuity $x_{j}$. In this case, instead of (5), the SS-solution $\psi_{0}(x)$ should be defined piecewise on each continuity interval of $w_{0}(x)$, and it is by definition equal to zero at the discontinuities: $\psi_{0}\left(x_{j}\right)=0$ (see [12] for the details).

Suppose now that there exist $n$ different base functions $w_{1}(x), \ldots, w_{n}(x)$, such that

$$
\begin{aligned}
& w_{j}(x)=k_{j}+w_{-\infty, j}(x), \quad w_{-\infty, j}=O\left(|x|^{-2}\right) \quad \text { at } x \rightarrow-\infty, \\
& w_{j}(x)=-k_{j}+w_{\infty, j}(x), \quad w_{\infty, j}=O\left(|x|^{-2}\right) \quad \text { at } x \rightarrow \infty,
\end{aligned}
$$

where all reals $\left\{k_{1}, k_{2}, \ldots, k_{n}\right\}$ are different, and using these functions the potential $U(x)$ can be represented in $n$ different ways:

$$
U(x)=-w_{j}^{2}(x)-i w_{j}^{\prime}(x)+k_{j}^{2} \text { for } j=1,2, \ldots, n .
$$

In this situation, the potential $U(x)$ obviously features $n$ coexisting spectral singularities at wavenumbers $k_{j}, j=1,2, \ldots, n$. Hence, in order to find a potential with multiple spectral singularities, it is sufficient to find any function $w_{j}(x)$ in equation (8) . However, given one of the functions $w_{j}(x)$, one has only the SS $k_{j}$ given, while determination the others requires finding all base functions $w_{j}(x)$ yielding (8)

Naturally, by far not every potential $U(x)$ can be represented in the form (8)), and thus the functions $w_{j}(x), j=1, \ldots, n$ must be interrelated. The requirement for two different base functions, say $w_{j}$ and $w_{j+1}$, to generate the same potential $U(x)$ in the explicit form reads

$$
w_{j+1}^{2}(x)+i w_{j+1}^{\prime}(x)-k_{j+1}^{2}=w_{j}^{2}(x)+i w_{j}^{\prime}(x)-k_{j}^{2} .
$$

For existence of $n$ SSs, this relation must be satisfied for all $j=1, \ldots, n-1$.

Let us look for a sequence of functions $w_{j}(x)$, which is generated by the recurrence relation

$$
w_{j+1}(x)=w_{j}(x)+\chi_{j}(x), \quad j=1, \ldots, n-1,
$$

where the newly introduced functions $\chi_{j}(x)$ satisfy the asymptotic behavior

$$
\lim _{x \rightarrow \pm \infty} \chi_{j}(x)=\mp\left(k_{j+1}-k_{j}\right), \quad \lim _{x \rightarrow \pm \infty} \chi_{j}^{\prime}(x)=0,
$$


which follows from (6) -(77). For the sake of simplicity, in what follows we assume that all asymptotic values at infinities are approached fast enough, i.e. faster than $O\left(|x|^{-2}\right)$. Using (10) and (9) one can obtain two relations

$$
\begin{aligned}
& i \chi_{j}^{\prime}+\chi_{j}^{2}+2 w_{j} \chi_{j}+k_{j}^{2}-k_{j+1}^{2}=0, \\
& i \chi_{j}^{\prime}-\chi_{j}^{2}+2 w_{j+1} \chi_{j}+k_{j}^{2}-k_{j+1}^{2}=0
\end{aligned}
$$

from which $w_{j}(x)$ and $w_{j+1}(x)$ can be expressed through $\chi_{j}(x)$ :

$$
\begin{aligned}
& w_{j}=-\frac{\chi_{j}}{2}-\frac{i \chi_{j}^{\prime}+k_{j}^{2}-k_{j+1}^{2}}{2 \chi_{j}}, \\
& w_{j+1}=\frac{\chi_{j}}{2}-\frac{i \chi_{j}^{\prime}+k_{j}^{2}-k_{j+1}^{2}}{2 \chi_{j}} .
\end{aligned}
$$

The obtained relations (14) and (15) already solve the problem of finding a potential with two spectral singularities, i.e. for the case $n=2$ and $j=1$. Indeed, by choosing any function $\chi_{1}(x)$ which has no real zeros, is two times differentiable, and satisfies the asymptotic requirements (11) with the prescribed $\mathrm{SSs} k_{1}$ and $k_{2}$, one readily obtains functions $w_{1}(x)$ and $w_{2}(x)$, and hence the potential $U(x)$. In fact, the formulated requirements on $\chi_{1}(x)$ can be weakened, and $\chi_{1}$ may be allowed to have a real zero $x_{0}$, provided that either $\chi_{1}^{\prime}\left(x_{0}\right)=i\left(k_{1}^{2}-k_{2}^{2}\right)$ or $\chi_{1}^{\prime}\left(x_{0}\right)=i\left(k_{1}^{2}-k_{2}^{2}\right) / 3$. In the former case, the singularity of functions $w_{1,2}$ at $x_{0}$ is removable, since using the l'Hôpital's rule one computes $\lim _{x \rightarrow x_{0}} w_{1,2}=\chi_{1}^{\prime \prime}\left(x_{0}\right) /\left(2\left(k_{2}^{2}-k_{1}^{2}\right)\right)$. In the latter case, both functions $w_{1,2}$ have singularities of the form $i /\left(x-x_{0}\right)$. Nevertheless, the potential $U(x)$ remains well-behaved around $x_{0}$, because singular terms in $w_{j}^{2}$ and $w_{j}^{\prime}$ cancel each other (here $j=1,2)$. As shown in [12] and mentioned above in this section, a singularity of function $w_{j}$ at $x_{0}$ corresponds to a zero of the corresponding SS solution: $\psi_{j}\left(x_{0}\right)=0$.

Additionally, the potential $U(x)$ remains well-behaved if the function $\chi_{1}(x)$ has a singularity of the form $\pm i /\left(x-x_{0}\right)$, where $x_{0}$ is any point of the real axis. More precisely, if $\chi_{1}$ has a singularity $i /\left(x-x_{0}\right)$, then function $w_{1}(x)$ has no singularity at $x_{0}$, while $w_{2}$ diverges as $i /\left(x-x_{0}\right)$. Therefore, the SS-solution corresponding to the wavevenumber $k_{2}$ has a zero at $x=x_{0}$. Vice versa, if $\chi_{1}$ has a singularity $-i /\left(x-x_{0}\right)$, then the function $w_{1}(x)$ diverges as $i /\left(x-x_{0}\right)$, and the SS-solution with the wavevenumber $k_{1}$ has a zero at $x=x_{0}$, while the function $w_{2}(x)$ is well-behaved at $x_{0}$, and the SS solution corresponding to the wavevenumber $k_{2}$ is zero-free (provided that $x_{0}$ is the only singularity of $\chi_{1}$ ). The conditions on $\chi_{j}$ can be weakened further, by allowing multiple discontinuities of the first derivative on a finite set of isolated points of the real axis, which ensures weaker constraints imposed on the base functions [12].

If the number of SSs is more than two, the functions $\chi_{j}$ are not arbitrary, but must satisfy the following relations

$$
\chi_{j}-\frac{i \chi_{j}^{\prime}+k_{j}^{2}-k_{j+1}^{2}}{\chi_{j}}=-\chi_{j+1}-\frac{i \chi_{j+1}^{\prime}+k_{j+1}^{2}-k_{j+2}^{2}}{\chi_{j+1}} .
$$

If two such functions $\chi_{j}$ and $\chi_{j+1}$ are found, then one can construct a potential $U(x)$ featuring three SSs, since now $w_{j}(x), w_{j+1}(x)$ and $w_{j+2}(x)$ and obtained self-consistently. 
Thus our next step is finding the functions $\chi_{j}$ and $\chi_{j+1}$. To this end we introduce new functions $\nu_{j}(x)$ for $j=1, \ldots, n-2$ by the relations

$$
\chi_{j+1}(x)=\chi_{j}(x) / \nu_{j}(x),
$$

and by the asymptotic behavior

$$
\lim _{x \rightarrow \pm \infty} \nu_{j}(x)=\frac{k_{j}-k_{j+1}}{k_{j+1}-k_{j+2}} .
$$

From (16) we obtain

$$
i \chi_{j} \nu_{j}^{\prime}+\left(k_{j+1}^{2}-k_{j+2}^{2}\right) \nu_{j}^{2}+\left(k_{j+1}^{2}-k_{j}^{2}+\chi_{j}^{2}\right) \nu_{j}+\chi_{j}^{2}=0 .
$$

Thus by choosing some $\nu_{j}(x)$ satisfying the asymptotic behavior (18), one can, in principle, determine $\chi_{j}(x)$ by solving the quadratic equation (19). Due to the definition (17), this readily gives $\chi_{j+1}$, and subsequently the base functions $w_{j}, w_{j+1}$, and $w_{j+2}$. Any of these functions can be used to obtain the complex potential $U(x)$. This resolves the problem of constructing complex potentials having three spectral singularities.

For constructing the potentials with four or more spectral singularities, one has to continue the self-consistent procedure described above, and ensure that $\nu_{j+1}$ and $\nu_{j}$ are chosen such that the function $\chi_{j+1}$ obtained from (19) with $j \rightarrow j+1$, i.e. from the equation

$$
i \chi_{j+1} \nu_{j+1}^{\prime}+\left(k_{j+2}^{2}-k_{j+3}^{2}\right) \nu_{j+1}^{2}+\left(k_{j+2}^{2}-k_{j+1}^{2}+\chi_{j+1}^{2}\right) \nu_{j+1}+\chi_{j+1}^{2}=0
$$

coincides with that given by (17). To this end one has to find $\nu_{j}$ and $\nu_{j+1}$ solving the system (19), (20), and (17). So far we have not been able to solve this problem,

i.e. to find an algorithm of constructing Schrödinger operators with more than three SSs (which of course does not mean nonexistence of the respective complex potentials). Therefore now we turn to illustration of our approach considering potentials with two and three coexisting SSs.

\section{Two spectral singularities}

\subsection{Self-dual spectral singularity}

Starting with self-dual SSs, from (14) with $j=1$ and $k_{1}=-k_{2}$ we obtain

$$
w_{1}=-\frac{\chi_{1}^{2}+i \chi_{1}^{\prime}}{2 \chi_{1}}
$$

and in terms of the function $\chi_{1}$ the searched potential reads

$$
U(x)=\frac{3\left(\chi_{1}^{\prime}\right)^{2}-2 \chi_{1} \chi_{1}^{\prime \prime}-\chi_{1}^{4}}{4 \chi_{1}^{2}}+k_{1}^{2} .
$$

Simple symmetry considerations show that if $\chi_{1}(x)$ is an anti-parity-time (anti- $\mathcal{P} \mathcal{T}$ )symmetric function, $\chi_{1}(x)=-\chi_{1}^{*}(-x)$, then the potential $U(x)$ is $\mathcal{P} \mathcal{T}$ symmetric, i.e. $U(x)=U^{*}(-x)[18$. This recovers a known property of $\mathcal{P} \mathcal{T}$-symmetric potentials to feature self-dual SSs [7]. In a more general case of the function $\chi_{1}(x)$ not being anti- $\mathcal{P} \mathcal{T}$ symmetric, using (22) one can systematically obtain asymmetric potentials $U(x)$ with 
self-dual SSs for any $k_{1}$ given beforehand (particular examples of asymmetric potentials with self-dual SSs have been previously discussed in [12, 13, 14]).

Using this approach, one can easily construct complex potentials featuring two SSs, $n=2$, having several free parameters, i.e. being deformable. We illustrate this showing the potentials depending on two complex parameters $a_{0,1}$. As the generating function $\chi_{1}(x)$ we use

$$
\chi_{1}(x)=\left(k_{1}-k_{2}\right) \tanh x+i a_{0} \operatorname{sech}\left(x-a_{1}\right),
$$

where $a_{0} \neq 0$ and $a_{1}$ are free parameters. The resulting potential $U(x)$ is $\mathcal{P} \mathcal{T}$ symmetric if $a_{0}$ is real and $a_{1}=0$ and is asymmetric otherwise. Using computer algebra, for $k_{2}=-k_{1}$ we obtain the resulting potential in the form

$$
\begin{aligned}
& U(x)=k_{1}^{2}+\left\{k_{1}^{2} \operatorname{sech}^{4} x\left(3+4 \sinh ^{2} x-4 k_{1}^{2} \sinh ^{4} x\right)\right. \\
& +i a_{0} k_{1} \operatorname{sech} y\left[2 \tanh x \operatorname{sech}^{2} y-3 \tanh y \operatorname{sech}^{2} x\right. \\
& \left.-\tanh x\left(8 k_{1}^{2} \tanh ^{2} x+1-2 \operatorname{sech}^{2} x\right)\right] \\
& -\left(a_{0}^{2} / 4\right) \operatorname{sech}^{2} y\left(1+\operatorname{sech}^{2} y-24 k_{1}^{2} \tanh ^{2} x\right) \\
& \left.+2 i a_{0}^{3} k_{1} \tanh x \operatorname{sech}^{3} y-\left(a_{0}^{4} / 4\right) \operatorname{sech}^{4} y\right\} / \chi_{1}^{2}(x),
\end{aligned}
$$

where for the sake of brevity we have introduced $y=x-a_{1}$.

In order to illustrate the above results using examples of the scattering data of the constructed potentials, we employ the transfer matrix formalism, introducing a pair of left (superscript "L") and right (superscript "R") Jost solutions which for real $k$ are defined uniquely by their asymptotic behaviors

$$
\begin{array}{lll}
\phi_{1}^{\mathrm{L}}(x ; k) \rightarrow e^{i k x}, & \phi_{2}^{\mathrm{L}}(x ; k) \rightarrow e^{-i k x} & \text { at } x \rightarrow-\infty, \\
\phi_{1}^{\mathrm{R}}(x ; k) \rightarrow e^{i k x}, & \phi_{2}^{\mathrm{R}}(x ; k) \rightarrow e^{-i k x} & \text { at } x \rightarrow+\infty .
\end{array}
$$

Since a scattering solution $\psi(x)$ of the Schrödinger equation (11) is a linear combination of the Jost solutions: $\psi(x)=a^{\mathrm{L}} \phi_{1}^{\mathrm{L}}+b^{\mathrm{L}} \phi_{2}^{\mathrm{L}}=a^{\mathrm{R}} \phi_{1}^{\mathrm{R}}+b^{\mathrm{R}} \phi_{2}^{\mathrm{R}}$, one obtains the relation between the coefficients $a^{\mathrm{L}, \mathrm{R}}, b^{\mathrm{L}, \mathrm{R}}$ defining the transfer matrix $M(k)$ :

$$
\left(\begin{array}{c}
a^{\mathrm{R}} \\
b_{\mathrm{R}}
\end{array}\right)=M(k)\left(\begin{array}{c}
a^{\mathrm{L}} \\
b_{\mathrm{L}}
\end{array}\right), \quad M(k)=\left(\begin{array}{cc}
M_{11}(k) & M_{12}(k) \\
M_{21}(k) & M_{22}(k)
\end{array}\right) .
$$

Real zeros of the matrix element $M_{22}(k)$ are SSs. Left and right transmission and reflection coefficients are computed as

$$
T^{\mathrm{L}}=T^{\mathrm{R}}=T=\frac{1}{M_{22}}, \quad R^{\mathrm{L}}=-\frac{M_{21}}{M_{22}}, \quad R^{\mathrm{R}}=\frac{M_{12}}{M_{22}} .
$$

Below we consider the scattering data $T(k), R^{\mathrm{L}}(k)$ and $R^{\mathrm{R}}(k)$ formally for both, positive and negative wavenumbers $k$, which naturally affects their physical interpretation (which is more conventionally is applied to positive $k$ ). A positive SS $k>0$ is associated with a lasing solution, while a negative SS $k<0$ describes a CPA solutions.

Once the potential $U(x)$ is given in an analytical form, the transfer matrix and the scattering characteristics can be computed numerically. In figure 1 we illustrate the shape of the potential by (24) for two combinations of free parameters $a_{0,1}$ which 

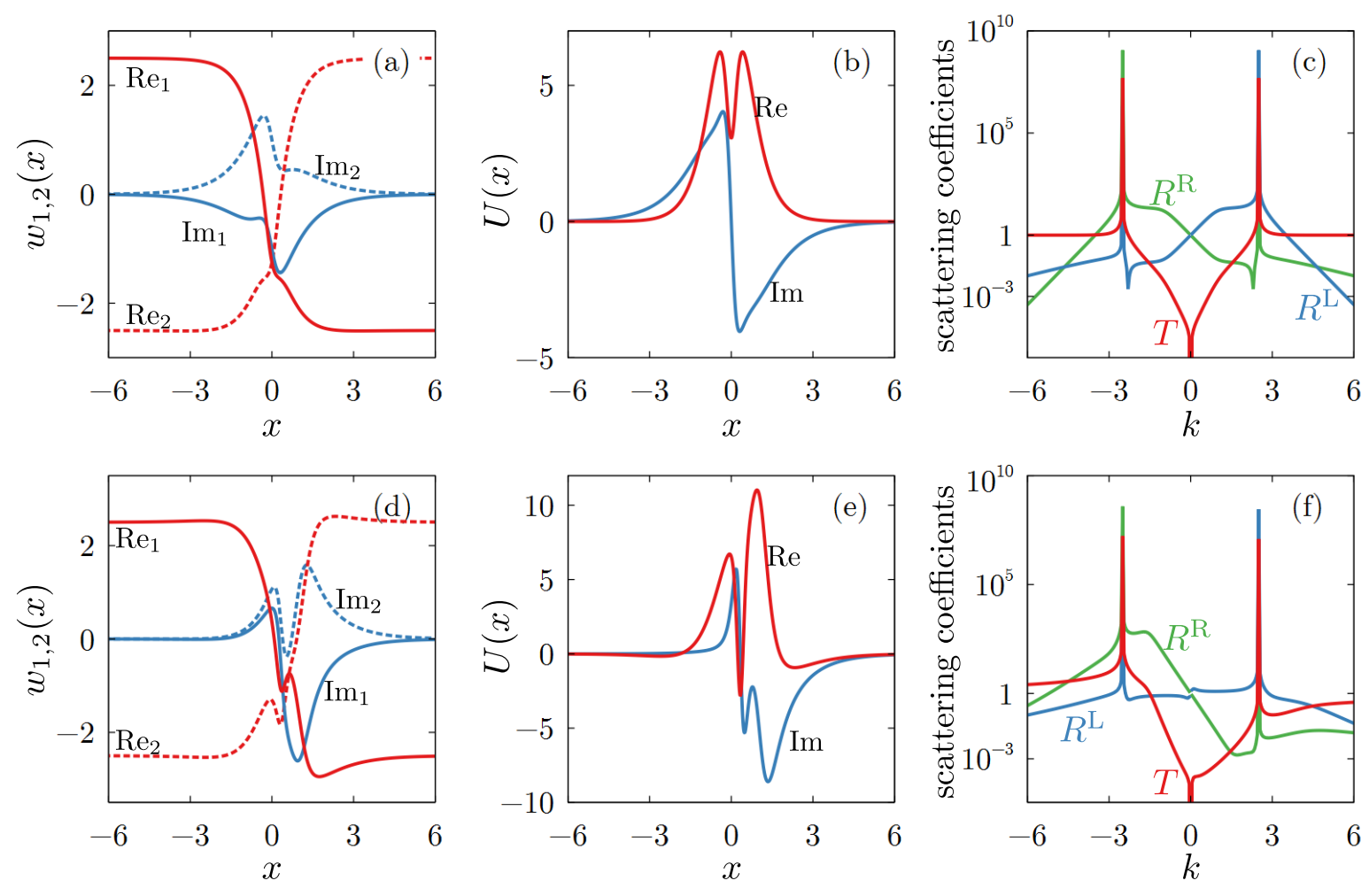

Figure 1. Examples of $\mathcal{P} \mathcal{T}$-symmetric at $a_{0}=2, a_{1}=0(\mathrm{a}-\mathrm{c})$ and asymmetric at $a_{0}=2+i, a_{1}=1-i(\mathrm{~d}-\mathrm{f})$ potentials (24) with a self-dual SS at $k_{1}=-k_{2}=2.5$. Panels $(\mathrm{a}, \mathrm{d})$ show real and imaginary parts of the functions $w_{1}(x)$ (solid lined) and $w_{2}(x)$ (dashed lines). Panels (b,e) show real and imaginary parts of potentials $U(x)$. In (a), (b), (e) and (d) the red and blue curves are the real (Re) and imaginary (Im) parts, respectively. Panels (c,f) show moduli of the transmission coefficient $T$ (red curves) and left and right reflection coefficients $R^{\mathrm{L}, \mathrm{R}}$ (blue and green curves, respectively).

correspond to the $\mathcal{P} \mathcal{T}$-symmetric case [figure $\mathbb{T}(a, b)]$ and to the asymmetric case [figure 1(d,e)] and for $k_{1}=-k_{2}=2.5$ in either case. In the right column we show the

numerically computed scattering characteristics for the respective potentials [figure 1 (c) and (f) for symmetric and asymmetric cases]. In accordance with the analytical results, the moduli of scattering coefficients for both potentials feature two extremely sharp peaks at $k_{1}$ and $k_{2}$, illustrating self-duality of the SS.

\subsection{Two independent spectral singularities}

Now we dismiss the requirement of self-duality and present an example of a complex potential with spectral singularities at any two wavevectors $k_{1,2}$ chosen beforehand. We use the two-parametric family given by equation (23) with $k_{1} \neq-k_{2}$. Substituting (23) into (14) and (15) with $j=1$, one can find explicitly the base functions $w_{1,2}(x)$ and recover the potential $U(x)$ in the analytical form. Since the resulting analytical expressions are rather bulky, we do not present them in the text and limit the presentation by plotting functions $w_{1,2}$ and potential $U(x)$ in figure 2. This figure 

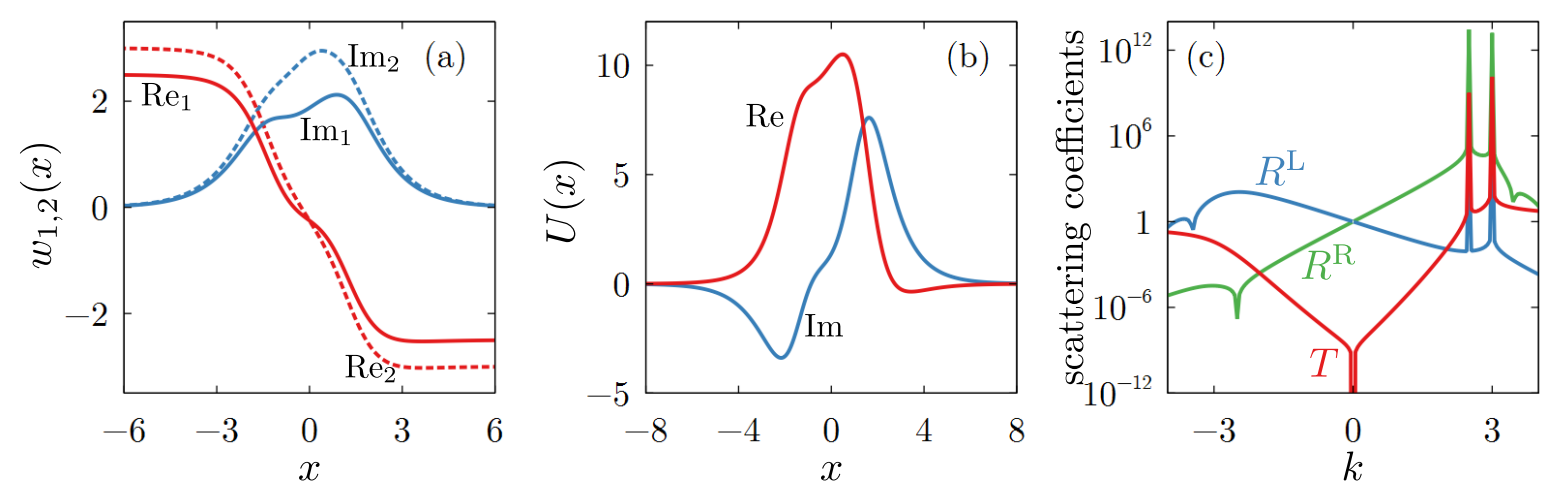

Figure 2. Example of a potential with two independent SSs at $k_{1}=2.5$ and $k_{2}=3$. The functions $w_{1,2}$ and the potential $U(x)$ are obtained using (23) with $a_{0}=1$ and $a_{1}=0$. The panels are organized in the same way as those in figure 1 $(\mathrm{a}, \mathrm{b}, \mathrm{c})$.
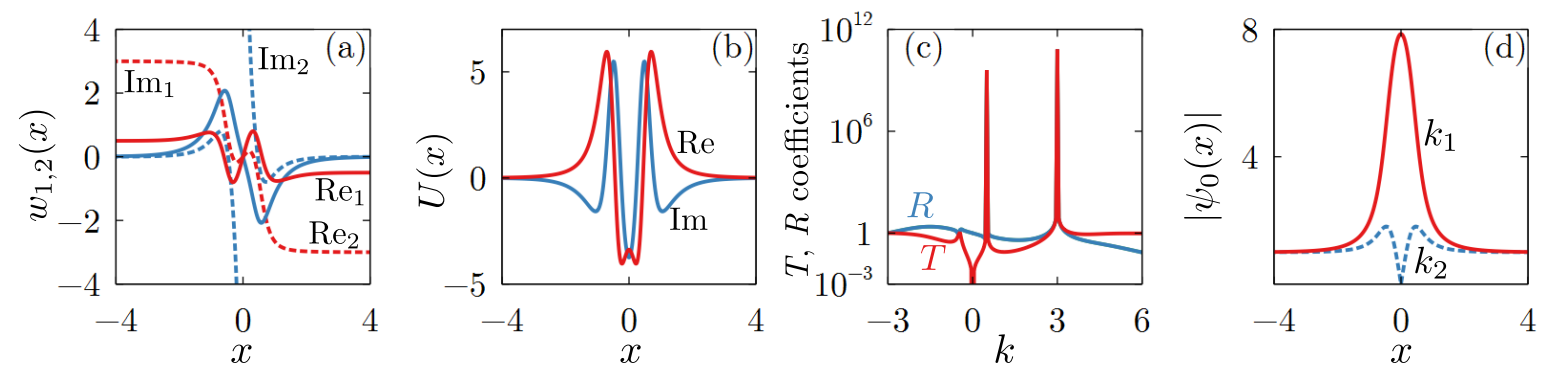

Figure 3. Example of a potential with two independent SSs at $k_{1}=0.5$ and $k_{2}=3$ one of which corresponds to a SS-solution with a zero. Functions $w_{1,2}$ and the potential $U(x)$ are obtained using (28). Panels (a-c) are organized in the same way as those in figure 2 (the potential is even function, hence moduli of left and right reflection coefficients are equal), and (d) shows amplitudes of SS-solutions associated with $k_{1}$ and $k_{2}$ (solid red line and dotted blue line, respectively).

illustrates the situation when the potential lases at two different wavevenumbers: $k_{1}=$ 2.5 and $k_{2}=3$. Notice that the constructed SSs are not self-dual, i.e. the corresponding scattering coefficients peaks do not have counterparts in the negative $k$ half-axis. Potentials with spectral singularities at any other combinations of wavevenumbers can be also constructed easily.

In the above examples the SS-solutions do not vanish on the real axis. In order to construct a potential enabling a SS solution with a real zero, we explore the function $\chi_{1}(x)$ having a singularity at $x=0$ :

$$
\chi_{1}(x)=\left(k_{1}-k_{2}\right) \tanh x+i \frac{\operatorname{sech} x}{x} .
$$

The respective complex potential $U(x)$ is a symmetric (even) function. A representative example of such a potential is shown in figure 3. In agreement with the discussion in section 2, the SS solution associated with the wavenumber $k_{1}$ is zero-free, whereas that with $k_{2}$ has a zero at $x=0$. Without loss of generality, both shown SS solutions plotted in Fig. [3](d,e) are normalized such that $\lim _{x \rightarrow \pm \infty}|\psi|=1$. 

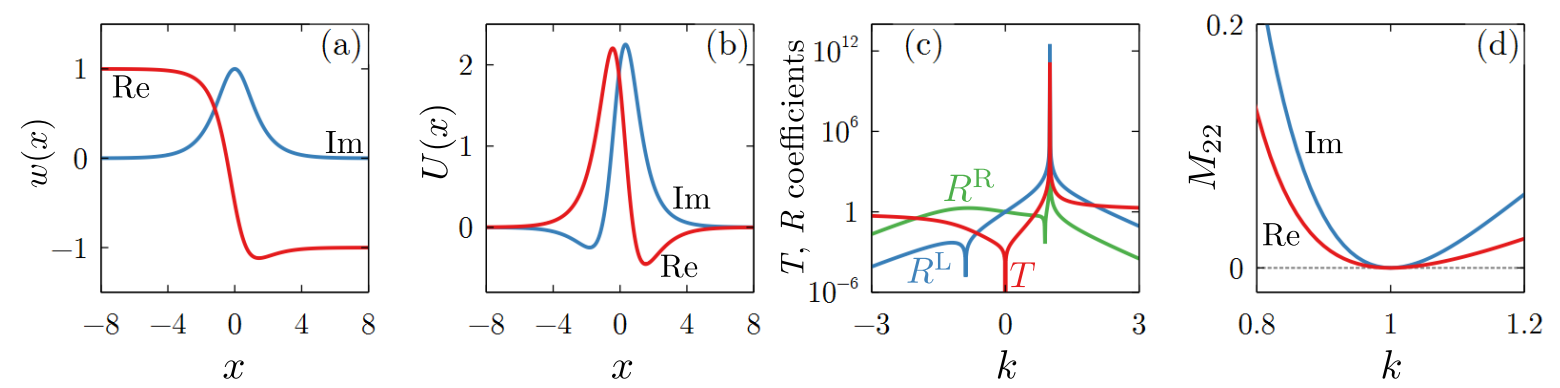

Figure 4. Potential (32) with a second-order SS. (a,b) show function $\tilde{w}(x)$ and corresponding potential $\tilde{U}(x)$; real and blue curves correspond to real (Re) and imaginary (Im) parts, respectively. (c) and (d) show moduli of scattering coefficients and real and imaginary parts of transfer matrix element $M_{22}(k)$ (the latter are plotted only in the vicinity of the SS). In all panels $k_{1}=1$.

\section{A second-order spectral singularity from the collision of two first-order ones}

Our approach to construction of multiple coexisting SSs proves to be useful also for designing potentials with a higher-order SS. In this section we solve the problem of creating a second-order SS by intentionally colliding two simple SSs $k_{1}$ and $k_{2}$ (i.e. implementing the mechanism inverse to the splitting of a second-order SS considered previously [11, 15]). To this end, we rewrite relations (14)-(15) with $j=1$, but make the dependence on $k_{1,2}$ explicit:

$$
\begin{aligned}
& w_{1}\left(x ; k_{1}, k_{2}\right)=-\frac{\chi_{1}\left(x ; k_{1}, k_{2}\right)}{2}-\frac{i \frac{\partial \chi_{1}\left(x ; k_{1}, k_{2}\right)}{\partial x}+k_{1}^{2}-k_{2}^{2}}{2 \chi_{1}\left(x ; k_{1}, k_{2}\right)}, \\
& w_{2}\left(x ; k_{1}, k_{2}\right)=\frac{\chi_{1}\left(x ; k_{1}, k_{2}\right)}{2}-\frac{i \frac{\partial \chi_{1}\left(x ; k_{1}, k_{2}\right)}{\partial x}+k_{1}^{2}-k_{2}^{2}}{2 \chi_{1}\left(x ; k_{1}, k_{2}\right)} .
\end{aligned}
$$

Let $k_{1}$ be fixed and $k_{2}$ approach $k_{1}$. We look for the situation when two SSs collide and result in the identical SS solution, i.e., $\lim _{k_{2} \rightarrow k_{1}} w_{1}=\lim _{k_{2} \rightarrow k_{1}} w_{2}$. This is possible if $\lim _{k_{2} \rightarrow k_{1}} \chi_{1}=\lim _{k_{2} \rightarrow k_{1}} \frac{\partial \chi_{1}}{\partial x}=0$. Then we can use the l'Hôpital's rule to compute

$$
\tilde{w}(x):=\lim _{k_{2} \rightarrow k_{1}} w_{1}=\lim _{k_{2} \rightarrow k_{1}} w_{2}=-\lim _{k_{2} \rightarrow k_{1}} \frac{i \frac{\partial^{2} \chi_{1}\left(x ; k_{1}, k_{2}\right)}{\partial k_{2} \partial x}-2 k_{2}}{2 \frac{\partial \chi_{1}\left(x ; k_{1}, k_{2}\right)}{\partial k_{2}}} .
$$

If the latter limit exists, then the potential $\tilde{U}(x)=-\tilde{w}^{2}-i \tilde{w}^{\prime}+k_{1}^{2}$ is expected to feature a second-order SS.

As a simple example, consider (23) with $a_{0}=k_{1}-k_{2}$ and $a_{1}=0$. From (31) we compute the limiting function $\tilde{w}(x)=-k_{1} \tanh x-\frac{1}{2} \operatorname{sech} x+i k_{1} \operatorname{sech} x$ which generates 
the potential in the form

$$
\tilde{U}(x)=\left(2\left(k_{1}+\frac{i}{2}\right)^{2}(1+i \sinh x)+\frac{1}{4}\right) \operatorname{sech}^{2} x .
$$

This potential and its scattering diagnostics are illustrated in figure 4. Both real and imaginary parts of the transfer matrix coefficient $M_{22}(k)$ remain nonnegative in the neighborhood of the spectral singularity, which corroborates that the latter is of the second order.

\section{Three spectral singularities and higher-order spectral singularities in pseudo-Hermitian Schrödinger operators}

A particularly interesting situation emerges if one of the base functions $w_{1}$ or $w_{2}$ is realvalued. In this situation the potential $U(x)$, even if it is not $\mathcal{P} \mathcal{T}$ symmetric, features a special antilinear symmetry [19] which enables self-dual SSs [12. To be specific, if $w_{1}$ is real valued, then for all real $k$ the diagonal elements of the transfer matrix of the potential $U(x)$ satisfy the identities [12]

$$
M_{11}(k)=M_{22}^{*}(k) \frac{k+k_{1}}{k-k_{1}}, \quad M_{22}(k)=M_{11}^{*}(k) \frac{k-k_{1}}{k+k_{1}} .
$$

(If $w_{2}$ is real-valued, then in identities (33) $k_{1}$ should be replaced with $k_{2}$ ). Thus there is always a SS at $k_{1}$. Moreover, using the identity $M_{11}(k)=M_{22}(-k)$ which holds for all real $k$, we deduce that any eventual SS at $k$ different from $k_{1}$ is always self-dual. Therefore, depending on the combination of $k_{1}$ and $k_{2}$, two different situations can be realized:

(i) If $k_{2}=-k_{1}$, then there is a self-dual SS at $\pm k_{1}$. However, this SS is distinctively different from self-dual SSs in section 3.1 because in the present case SS at $k_{1}$ is at least of the second order, whereas the opposite spectral singularity at $-k_{1}$ is generically of the first order. Indeed, by construction $M_{22}\left(k_{1}\right)=0$ and $M_{22}\left(-k_{1}\right)=0$. Hence $M_{11}\left(k_{1}\right)=0$, and, using relations (333), we readily observe that $M_{22}(k)=O\left(\left(k-k_{1}\right)^{2}\right)$ as $k \rightarrow k_{1}$.

(ii) If $k_{2}$ is different from $-k_{1}$, then the potential $U(x)$ features three SSs, two of which correspond to the self-dual SS at $\pm k_{2}$, and the third one is at $k_{1}$. (These SSs will be generically of the first order).

In order to formulate the condition for function $w_{1}$ to be real-valued, we represent $\chi_{1}=\rho(x) e^{i \phi(x)}$, where $\rho$ and $\phi$ are real functions, and from Eq. (14) with $j=1$ we obtain that imaginary part of $w_{1}$ disappears if

$$
\sin \phi=\frac{\rho^{\prime}}{k_{1}^{2}-k_{2}^{2}-\rho^{2}} .
$$

Thus, starting with some function $\rho(x)$ which has asymptotic behavior $|\rho| \rightarrow\left|k_{1}-k_{2}\right|$ as $x \rightarrow \pm \infty$, is differentiable twice and smooth enough to ensure $\left|\rho^{\prime} /\left(k_{2}^{2}-k_{1}^{2}+\rho^{2}\right)\right| \leq 1$ for all $x \in \mathbb{R}$, one can, in principle, recover the corresponding phase $\phi$ and then obtain 
function $\chi_{1}(x)$ and real-valued function $w_{1}(x)$. However, the procedure is not completely straightforward, because (34) defines only sine of $\phi$ but does not ensure the required asymptotic behaviour of $\chi_{1}$ at the infinities. Meantime, the requirement for $\arg \chi_{1}$ to change from 0 to $\pi$ may result in discontinuities of the first kind of $\chi_{1}$. In order to illustrate how these problems can be overcome, we explore the substitution for $\rho$ in the form of an odd function with a discontinuity of the second kind at $x=0$ :

$$
\rho=\frac{1}{x\left(x^{2}+1\right)}-\left(k_{2}-k_{1}\right) \tanh (a x) .
$$

Here $x^{2}+1$ is incorporated in the denominator in order to speed up the algebraic decay at infinities, and $a>0$ is a free parameter. Considering the behavior around the discontinuity at the origin, using (34) we observe that $\lim _{x \rightarrow 0} \sin \phi(x)=1$. Since $\rho(x)$ is an odd function of $x$, the required asymptotic behavior of $\chi_{1}(x)$ at the infinities is achieved if we fix $\cos \phi=\sqrt{1-\sin ^{2} \phi}$. As explained in section 2, for the resulting potential $U(x)$ to be well-behaved, in the vicinity of the discontinuity $\chi_{1}$ must behave as $i / x$, i.e., real part of $\chi_{1}$ must tend to zero as $x$ approaches the origin. This leads to the conditions $\cos \phi(0)=d[\cos \phi(0)] / d x=0$, i.e., $d^{2}[\sin \phi(0)] / d x^{2}=0$, which in its turn imposes an additional relation between the wavenumbers $k_{1,2}$ and the free parameter $a$ :

$$
\left(k_{1}-k_{2}\right)\left(k_{1}+k_{2}-3 a\right)+3=0 .
$$

In order to illustrate a higher-order SS [the above case (i)], we choose $a=1$ and $k_{1}=-k_{2}=1 / 2$. The obtained results are illustrated in Fig. 5. In Fig. 5)(a) we observe that function $w_{1}$ is real-valued, whereas function $w_{2}$ has nontrivial real and imaginary parts, the latter with a discontinuity $i / x$. The potential $U(x)$, which is an even, continuous and smooth function, is plotted in figure 5 (b). It decays as $\propto x^{-3}$ as $x \rightarrow \pm \infty$. As shown in figure 5 (c), scattering coefficients feature sharp resonances at $\pm 1 / 2$ which is a manifestation of the self-dual SS. At the same time, plotting real and imaginary part of the transfer matrix coefficient $M_{22}(k)$ in figure $5(\mathrm{f})$, we observe that spectral singularities at $k_{1}=1 / 2$ and $k_{2}=-1 / 2$ are distinctively different: the former is of the second order (real and imaginary parts of $M_{22}(k)$ remain negative in the vicinity of the SS, except for the very point of SS where they are both zero), and the latter is of the first order. In addition, in figure. 5(d,e) we show amplitudes and phases of the lasing solution at $k_{1}$ and the CPA-solution at $k_{2}$. The laser solution is constant-amplitude due to the fact that $w_{1}$ is real valued, and the CPA solution has a zero at $x=0$ which agrees with the previous discussion on the singularity of function $w_{2}$.

In order to provide an explicit example for the case (ii) with coexisting self-dual and simple spectral singularities, we choose $a=1, k_{1}=5 / 2$ and $k_{2}=-1 / 2$. The resulting functions $w_{1,2}$, localized potential $U(x)$, and moduli of the scattering coefficients are plotted in figure 6. According to the analytical prediction, the potential features a spectral singularity (laser) at $k_{1}$ and a pair of spectral singularities at $\pm k_{2}$ which correspond to laser and CPA modes. 

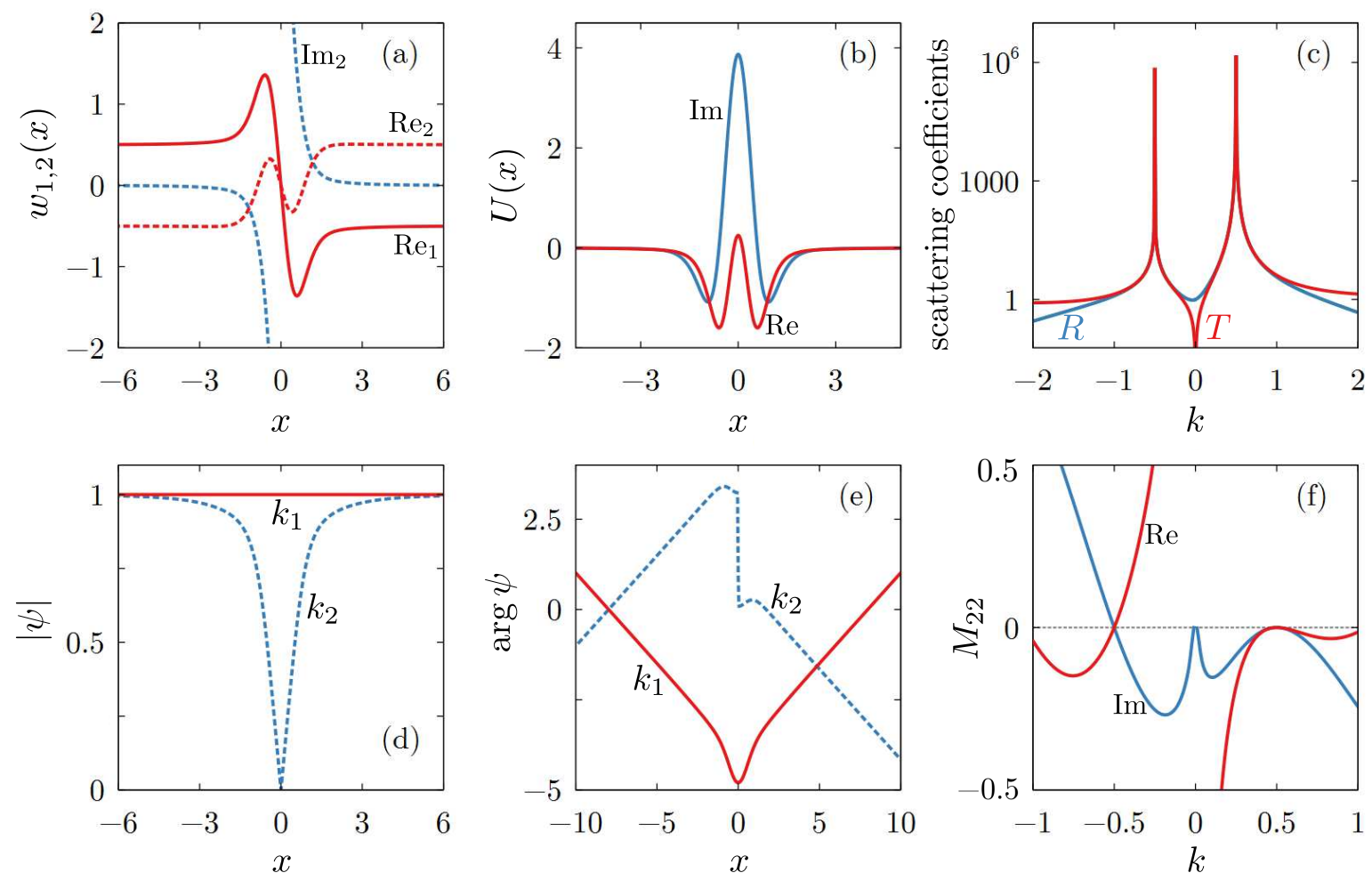

Figure 5. Example of a pseudo-Hermitian potential which features an "unusual" selfdual SS consisting of a first-order SS with $k>0$ and a second-order SS at $k<0$. (a) Real-valued function $w_{1}(x)$ and complex-valued function $w_{2}$ which generate a complex-valued potential $U(x)$ are shown in (b). Moduli of transmission and reflection coefficients (c) and real and imaginary parts of the transfer matrix entry $M_{22}(k)$ (f). Since the potential is an even functions, moduli of the left and right reflection coefficients are equal. Panels (d,e) show amplitudes and unwrapped radian arguments (i.e. phases) of the constant-amplitude laser solution corresponding to the SS at $k_{1}=1 / 2$ and the CPA solution corresponding to the SS at $k_{2}=-1 / 2$.

\section{Three independent spectral singularities}

Now we address a general case where three spectral singularities are achieved at some arbitrarily prescribed wavenumbers without any assumption of self-duality. According to equation (19), the solution to this problem is reduced to solving a quadratic equation with respect to $\chi_{1}$ with the auxiliary function $\nu_{1}(x)$ chosen to satisfy the asymptotic behavior (18). Once $\chi_{1}(x)$ is known, the base function $w_{1}$ and the full potential $U(x)$ can be determined. The most challenging issue is that not any choice of $\nu_{1}$ leads to a "suitable" solution of the quadratic equation. For example, for a naive choice of $\nu_{1}$ in the form of a purely real function or an anti- $\mathcal{P} \mathcal{T}$-symmetric one, even chosen to satisfy asymptotic requirements (18), the generated $\chi_{1}$ can be discontinuous or singular. Nevertheless, it is possible to make a suitable choice of $\nu_{1}$. For an explicit example, let us choose $k_{1,2,3}=1,2,3$, i.e. we look for a potential that lases at three different wavenumbers, and explore the following substitution which sufficiently rapidly 

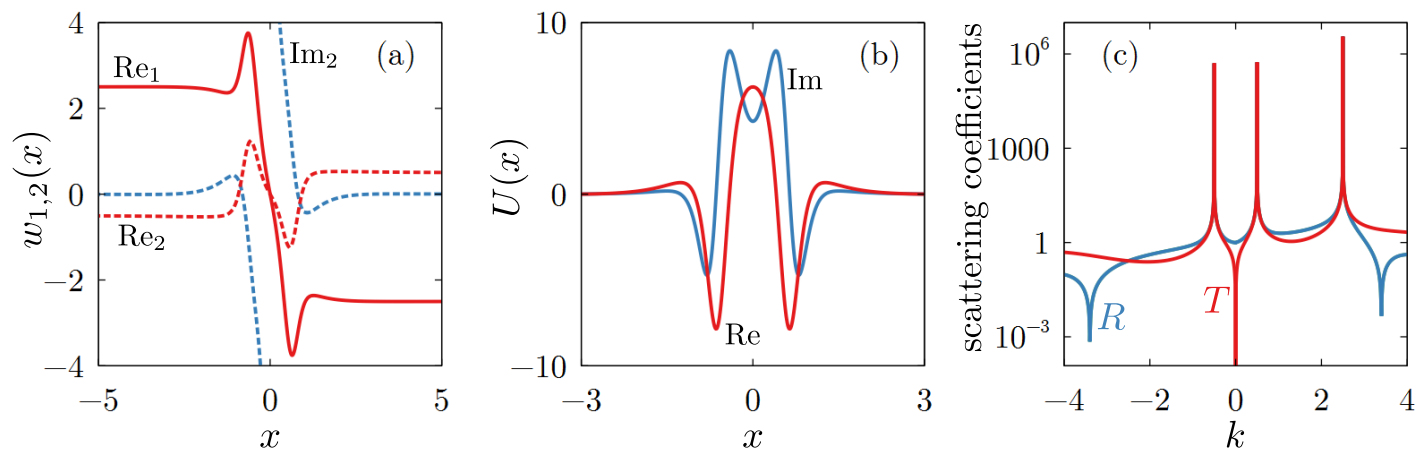

Figure 6. Example of a pseudo-Hermitian potential which features three SS, two of which form a self-dual SS. (a) Real-valued function $w_{1}(x)$ and complex-valued function $w_{2}$ which generate a complex-valued potential $U(x)$ shown in (b). (c) Moduli of the scattering coefficients; the left and right reflection coefficients are equal. Three peaks at $k= \pm 1 / 2$ and $k=5 / 2$ correspond to a self-dual SS $k= \pm 1 / 2$ coexisting with a simple SS at $k=5 / 2$.
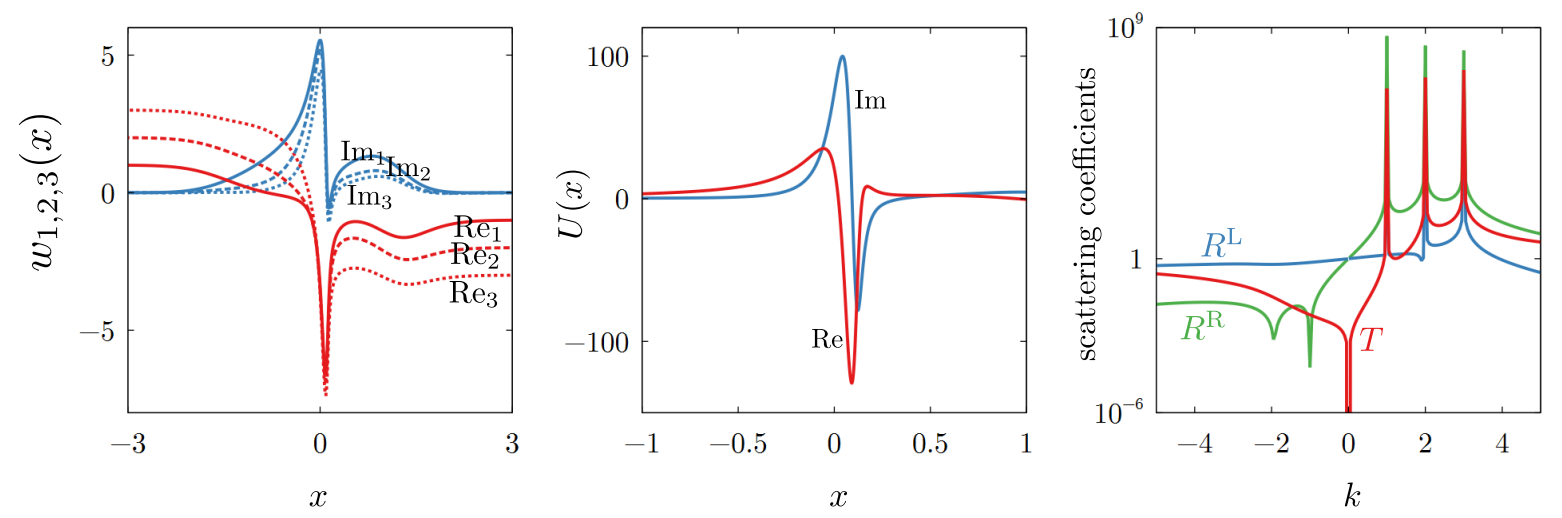

Figure 7. Example of a potential with three spectral singularities obtained according to our procedure using the substitution (37) with $k_{1,2,3}=1,2,3$ and $z=-1 / 2-i / 10$. Panels (a) shows real (Re) and imaginary (Im) parts of base functions $w_{1,2,3}(x)$. Panel (b) shows real and imaginary parts of the resulting potential, and (c) shows the moduli of the scattering coefficients.

approached the asymptotic behavior (18):

$$
\nu_{1}=\frac{k_{1}-k_{2}}{k_{2}-k_{3}}+(i x+z) e^{-x^{2}},
$$

where $z$ is a complex parameter. Three base functions $w_{1,2,3}(x)$ obtained for $z=$ $-1 / 2-i / 10$ and the resulting potential $U(x)$ are presented in figure $7(\mathrm{a}, \mathrm{b})$. Plots of moduli of the scattering coefficients in figure 7 (c) clearly indicate three coexisting resonant peaks which validate the result. 


\section{Conclusion}

In this paper we have described an algorithmic approach for designing complex potential resulting in simultaneous emergence of two and three SSs in the spectra of the respective Schrödinger operators. The construction is based on the universal form of the potential generated by a base function: different SS solutions are constructed using different base functions, while all these base functions result in the same complex potential. Using this approach, we have been able to construct potentials that result in two and three spectral singularities. A procedure for construction of potentials that feature secondorder spectral singularities has been also elaborated. In the situation, when the potential is pseudo-Hermitian, we have implemented a situation when one simple and one self-dual SS coexist in the spectrum, and when a self-dual SS consists of a first- and a secondorder SSs. In the meantime, a systematic procedure of obtaining complex potentials featuring any number of SSs in their spectra at different wavelengths remains an open question.

\section{Acknowledgments}

VVK is grateful to Z. Ahmed for the comments stimulated this work. VVK acknowledges financial support from the Portuguese Foundation for Science and Technology (FCT) under Contract no. UIDB/00618/2020. The work of DAZ was supported by the Foundation for the Advancement of Theoretical Physics and Mathematics "BASIS" (Grant No. 19-1-3-41-1).

\section{References}

[1] Naimark M A 1954 Investigation of the spectrum and the expansion in eigenfunctions of a nonselfadjoint operator of the second order on a semi-axis Tr. Mosk. Mat. Obs. 3 181-270 Schwartz J 1960 Some non-selfadjoint operators Comm. Pure Appl. Math. 13609

Vainberg B 1968 On the analytical properties of the resolvent for a certain class of operatorpencils Math. USSR Sbornik 6 241-273

[2] Khapalyuk A P 1962 Dokl. Akad. Nauk BelSSR 6, 301 (in Russian) Khapalyuk A P 1982 Opt. Spectrosk. 52194 Zharov A A and Zaboronkova T M 1983 Fiz. Plazmy 9995 (in Russian) Zharov A A and Kotov A K 1984 Fiz. Plazmy 10615 (in Russian)

Poladian L 1996 Resonance mode expansions and exact solutions for nonuniform gratings Phys. Rev. E 542963

[3] Chong Y D, Ge L, Cao H and Stone A D 2010 Coherent Perfect Absorbers: Time-Reversed Lasers Phys. Rev. Lett. 105053901

[4] Mostafazadeh A 2009 Spectral Singularities of Complex Scattering Potentials and Infinite Reflection and Transmission Coefficients at Real Energies Phys. Rev. Lett. 102220402

[5] Mostafazadeh A and Mehri-Dehnavi H 2009 Spectral singularities, biorthonormal systems and a two-parameter family of complex point interactions J. Phys. A: Math. Theor. 42125303

[6] Ahmed Z 2009 Zero width resonance (spectral singularity) in a complex PT-symmetric potential, J. Phys. A: Math. Theor. 42472005 
[7] Longhi S $2010 \mathcal{P} \mathcal{T}$-symmetric laser absorber Phys. Rev. A 82031801

[8] Wan W, Chong Y, Ge L, Noh H, Stone A D and Cao H 2011 Time-reversed lasing and interferometric control of absorption Science 331 889-892

[9] Baranov D. G, Krasnok A, Shegai T, Alú A and Chong Y D 2017 Coherent perfect absorbers: linear control of light with light Nature Reviews Materials 217064

Rosanov N N (2017) Antilaser: resonance absorption mode or coherent perfect absorption? Physics - Uspekhi 60818

[10] Mostafazadeh A 2014 A dynamical formulation of one-dimensional scattering theory and its applications in optics Ann. Phys. 341 77-85

Mostafazadeh A 2014 Unidirectionally invisible potentials as local building blocks of all scattering potentials Phys. Rev. A 90023833

[11] Konotop V V, Lakshtanov E, and Vainberg B 2019 Designing lasing and perfectly absorbing potentials Phys. Rev. A 99043838

[12] Zezyulin D A and Konotop V V 2020 A universal form of localized complex potentials with spectral singularities New J. Phys. 22013057

[13] Mostafazadeh A 2012 Self-dual spectral singularities and coherent perfect absorbing lasers without $\mathcal{P} \mathcal{T}$-symmetry J. Phys. A: Math. Theor. 45444024

[14] Konotop V V and Zezyulin D A 2017 Phase transition through the splitting of self-dual spectral singularity in optical potentials Opt. Lett. 425206

Konotop V V and Zezyulin D A 2019 Opt. Lett. 441051

[15] Hang C, Huang G, and Konotop V V 2016 Tunable spectral singularities: coherent perfect absorber and laser in an atomic medium New J. Phys. 1808500

[16] Ahmed Z 2012 New features of scattering from a one-dimensional non-Hermitian (complex) potential J. Phys. A: Math. Theor. 45032004

[17] Borisov D A and Zezyulin D A 2019 Spacing gain and absorption in a simple $\mathcal{P} \mathcal{T}$-symmetric model: spectral singularities and ladders of eigenvalues and resonances $J$ Phys. A: Math. Theor. 52445202

[18] Bender C M and Boettcher S 1998 Real Spectra in Non-Hermitian Hamiltonians Having $\mathcal{P} \mathcal{T}$ Symmetry Phys. Rev. Lett. 805243

Bender C M 2007 Making sense of non-Hermitian Hamiltonians Rep. Prog. Phys. 70 947-1018

[19] Nixon S and Yang J 2016 All-real spectra in optical systems with arbitrary gain-and-loss distributions Phys. Rev. A 93 031802(R) 